\title{
Virtual Equipment Assembly Process Modeling and Application Based on Knowledge Representation
}

\author{
Lai Jiang ${ }^{1+}$ and Xiaokun Liu ${ }^{2}$ \\ ${ }^{1}$ School of Economics and Management, Northeast Electric Power University, Jilin, China \\ ${ }^{2}$ School of Automotive Engineering, Shanghai University Of Engineering Science, Shanghai, China
}

\begin{abstract}
To solve the problems of expressing the complex relationship between the current virtual equipment parts and the process flow and dull description to the assembly process in virtual equipment assembly process, the paper introduced knowledge representation method in production system into virtual equipment assembly process modeling. Using production system to describe the virtual equipment assembly process abstractly and normatively achieve the logic interconnection between different steps in assembly process. Arrange the virtual assembly process follow the calculation process of production system. This improves the flexibility and standardization of the practical application platform. Finally, it takes a multifunction machine tool in existing virtual system as an example to verify the method is efficient.
\end{abstract}

Keywords: virtual equipment assembly, production system, process modelling.

\section{Introduction}

Virtual reality technology has been widely used in many teaching and training system, and has gradually changing the traditional teaching process [1]. Because of the characteristics of electromechanical equipment itself, there are many limitations in practical teaching and training process of assemble or disassemble equipment, virtual equipment assembly teaching system can solve this kind of problem.

The design procedure used by equipment assembly training system generally first complete modelling of parts, display the assembly process according to the arranged steps and perform assembly process by reading parts model information in CAD system. As a supplement to practical training teaching, the virtual assembly system tend to focus on a high simulation of physical model, emphasizing effect of 3D modeling and standardize designing modeling flow, and simply or directly reproduce the virtual assembly process with a fixed step by programming. This method in a certain extent can meet the needs of teaching demonstration, but have more obvious shortcomings in strict simulating practice training. Reality equipment assembly process are more flexible and can be designed, such as many different paths can be chosen, some operation exist repetitive, operation path can be backtracking and reproduction, some steps are parallel and exist synchronization mechanism among them etc. In addition, the cases such as lack tools, supplies, personnel cooperation may occur during the assembly process. This requires virtual assembly system provides controllable and dynamic function to select and design assembly step, provide teaching cases design interface for teacher to simulate various situations during assembly process and assist teaching description and case analysis in practical training. Some complex modelling methods are used to express the equipment assembly process scientifically. Meta Fusion modeling method, proposed in reference [2], divided the whole operation process into several relatively independent, easy to be handled and common parts to build models separately. This method improves the versatility of modeling and worth reference. The MTN (Maintenance Task Net) emulative models mentioned in reference [3], using graphical method to build models, combining

\footnotetext{
+ Corresponding author.

E-mail address: hp2072@126.com
} 
the abstract maintenance task description with low-level activity simulation. The modeling method in reference [4], [5] is use semantic networks and Petri net knowledge representation. All of the above modeling methods have strong expression ability, symbol and standard is relatively mature, but they are more suitable for expressing assembly process of large equipment with complex logic and numerous parts. Equipment selected by virtual assembly practical teaching often has the characteristics of concise design and flexible assembly process, which generally need to explore and adjust the design case continuously. Meanwhile, taking into account the teacher's knowledge level and meeting the unit teaching needs, what descriptive problems must consider are the level of assemble or disassemble equipment and granularity partition.

The paper introduced the production system knowledge of artificial intelligence into assembly flow description of virtual equipment assembly practice teaching system. Production system implement process is very similar to human solving problems process. So it can be used to describe the process of assembly. Production system rules database standardize the assembly step, especially suitable for step by step teaching designed by teacher, and it greatly simplifies the development process of the teaching system. Perfectly designed production system can describe relations such as assembly sequence, choice, concurrency, conflict, repetition, synchronization at the semantic layer. As a kind of automated reasoning tools, production system can complete assembly demonstration teaching automatically at any time according to the present assembly equipment state, and give a reasonable standard of explanation. Separate production system knowledge database from reasoning machine can realize semantic interconnection between the knowledge resources of descript the assembly process and standardized organize of simulate assembly behavior. It can effectively solve the existing problems such as structure decomposition blank and model is too large, and greatly enhances the extensibility of the virtual equipment assembly teaching platform.

\section{Frame Design of the Virtual Assembly System Teaching Platform}

The scheme consists of the following four modules: human-computer interaction, virtual reality scene controlling, resource database, equipment assembly system. Figure 1 gives the system frame structure.

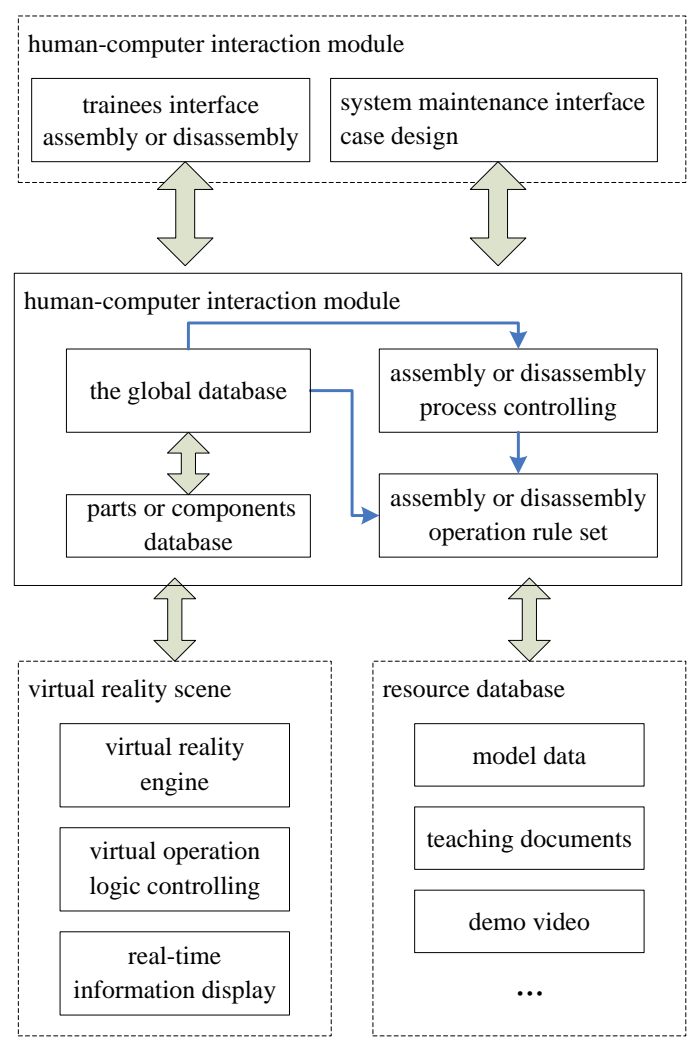

Fig. 1: Frame of teaching platform. 
Human-computer interaction uses interaction mechanism of human nature. Its main function is provides the virtual assembly interface for trainees. It can select the appropriate assembly operations, tools, parts and components according to the teacher's teaching design to complete assembly activities, real-time feedback operation result and prompts, provide teaching case design and maintenance interface for teachers. Equipment assembly system is the core part of the whole system which composition is the same as the structure of production system. It including four parts: the global database, assembly operation rule set and assembly process controlling module, parts or components database. The system mainly provides semantic level control for the entire system and import new equipment case by teacher's special maintenance interface. Virtual reality scene controlling module includes virtual reality engine, virtual operation logic module and real-time information display function. Virtual reality engine receive message from interactive module and equipment assembly system, read resource database, complete importing models, view settings, also real time calculate the data and instructions information according to the instruction of background operation logic module, and rendering the real time scene according to the processing result. Resource database system provides part model data, the demo video, teaching documents and other types of resource files. It complete related resource information maintenance through special interface, and in the practical application it store files into the server and provides cloud access interface.

\section{Modeling of Virtual Assembly Process}

The key problem the virtual assembly system should solve is to improve the simulate extent of virtual environment for meeting requirements of the design teaching case and achieve the effect of assist or partly replace physical assembly teaching and training. Therefore, the established virtual models must have a good fit degree with reality process and can meet the needs of design case flexibly and customize steps standardly. Existing virtual assembly models show that the design of general simplified model emphasizes designing the whole process with a single standard, that is, the process model is one-way solidification. The assembly process represented by program language, once identified, cannot be modified. It can't reach or meet the requirements of students explore study and teachers design teaching case. Some knowledge representation method had been widely used in many fields, such as semantic networks and Petri nets, etc. They have excellent modeling semantics and powerful modeling tools which were used to describe the complex system operation logic. But in terms of modeling that around training course, these methods are too complex and require high professional. It is not conducive to add the new knowledge that generated in the course of practice teaching into the system and gradually improve the level of teaching case. In the other hand, the program is complex in developing these process models by computer and difficult to develop and maintain. In this paper, the method based on production system was used to build modeling during assembly process. The method store the assembly process steps in form of knowledge rules which make it convenient, flexible and easy maintenance. Moreover, the realization of knowledge does not depend directly on the computer program which make it is convenient maintenance.

\subsection{Knowledge Representation Based on Production System}

Production system is proposed by American mathematician E.Post in 1943, as a transform theory of combination problem, the production is referred to symbolic transformation rules as similar to $\mathrm{A} \rightarrow \mathrm{Aa}$. Production System is mainly used in expert system of artificial intelligence and also means that realize knowledge representation methods like predicate formula by computer. Production system consists of a global database, production rules and control strategy as shown in Figure 2.

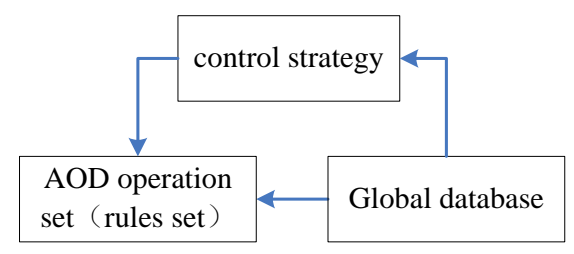

Fig. 2: Production system structure.

Global database is the main data structure used by production system and store all the information such as input facts, issues state and the problem to be solved, including intermediate results and the final results of 
reasoning. The data in the global database are the object that production rules process. Rules set is a knowledge collection of some domain represented in the form of rules and rules is represented by production. Rules set contains all transformation rules that convert the problem from the initial state to target state. The general form of rules is: "conditions" $\rightarrow$ "behavior" or "premise" $\rightarrow$ "conclusion". It is general expressed by program language as "if... Then...". The left side provides preconditions that the rule apply, the right side describe the behavior the rule applied or conclusion. When it determines premises or conditions of the rules, matching method is usually adopted. That is check the global database whether the situation the rule's premise or conditions pointed out could exist. If exist, the match is successfully, otherwise, failure. If the matching is successful, execute the actions specified by rules conduct or get the conclusion described by rules. Control strategy is the interpretation program of rules which specifies how to choose an available rule operates the global database, that is, it determines solving process or reasoning line of the problem. Typically, control strategy is responsible for matching production rule premise or condition with data in global database. It selected one rule from those successfully matched to running according to a certain strategy and ended the system running at the right time.

\subsection{Representation of Virtual Equipment Modeling Based on Production System}

(1) Representation of independent part. Independent part refers to those no detachable parts which is the smallest unit during assembly process. It can be abstracted to isolated facts and usually expressed in ternary (object, attribute, value). In the process of assembly, as the execution of the assembly action, independent parts appear constantly. In practical application, teacher can design their own independent parts according to the progress of teaching such as define component as an independent part.

(2) Representation of component. Component comes from a series of assembly operations on independent parts. It is non-terminal node of a tree structure formed in the process of operation positive (disassembly) or reverse (assembly). In production system, component is expressed as intermediate results and final result during assembly process in the global database. Component can be expressed by context. Existing multiple parts and components can be described in global database which can solve the describing problem of parallel operation. Setting conditions of parts and components for synchronously controlling assembly process and multi-persons' collaborative assembly . In practice, teacher can define the components (the key nodes) according to the teaching needs to strengthen knowledge points of training teaching.

(3) Representation of assembly operation. Disassembly operation is split operations to components. After disassembly, parts can be disassembled into some parts or components. In addition, after disassembly, components can be converted to another part state. The assembly operation is the reverse operation of disassembly operation, after assembling, parts or components can be new parts or another state. Assembly operations may require one or more tools to complete. As shown in Figure 3, assembly operation can be expressed in production rules which include the former and the latter. The former is the set of all the preconditions that the operation completed, including the existing tools, parts, components and the state description; the latter is the set of parts, components and the state description after finished assembly operation.

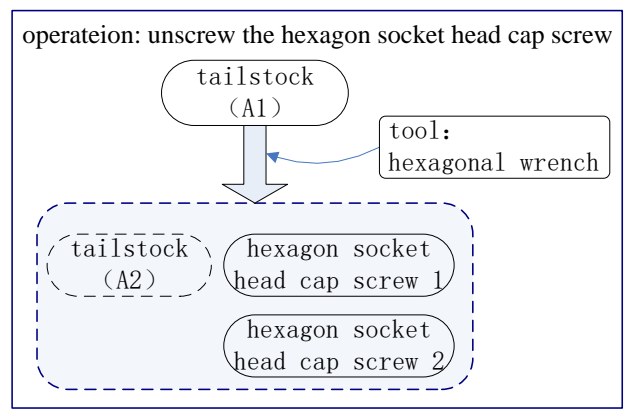

Fig. 3: Representation of assembly.

(4) Representation of assembly process. The process is the running (reasoning) process of production system. It's positive or reverse running corresponding to disassembly or assembly process. Disassembly process (positive) is starting from the existing components and gets disassembly results from the rules 
database. Assembly process (reverse) is starting from the existing parts, after continuously assemble operation through the rules database, the final result obtained. For students, the assembly process is a continuous exploring process in virtual environment, and any operation can be chosen. Combined with the characteristics of production system, according to the teaching demonstration or student needs, the system can also complete assembly process automatically by starting the control strategy of eliminate conflict up and select disassembly operation automatically from the rules database.

\section{Application}

According to the actual situation of electromechanical equipment assembly training courses and existing equipment in labs, some teaching cases have been developed in virtual assembly teaching system based on such equipment as Venus CQ90107 multi-function machine tools, multi-function machine tools, bench lathe and cylindrical gear reducer.

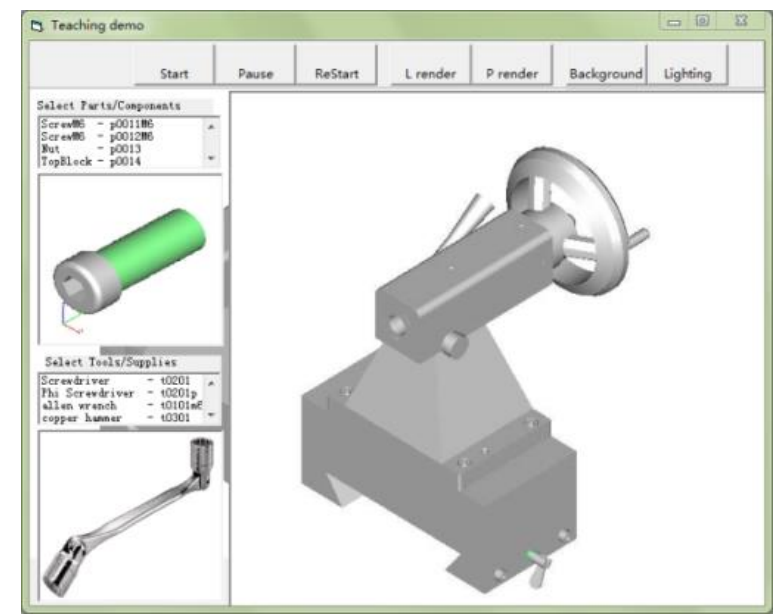

Fig. 4: Virtual assembly operation interface.

The develop process closely combined with teaching case. First, using CAD software to build 3D models of equipment parts and organizing related materials. Then on the basis of full argument, according to the method proposed in this paper and the assembly technical manual to build models during assembly process, describe corresponding assembly operations abstractly and write relative information into the system. According to the actual case, the trainees enter the system to complete the assembly operation in virtual environment. Feedback from training teacher and software development team shows that the modeling method is simple and flexible, clear and standard expression, greatly simplifies the modeling work and development process.

\section{Conclusions}

The method has been applied to the process of develop electromechanical equipment virtual assembly system and designed some teaching cases. The practice has shown that the method can effectively regulate the virtual assembly flow and solve the problem of using fixed program to describe the assembly process. This method is more efficient than other complex modeling method, especially suitable for developing teaching cases with small number of parts and lower complexity of assembly.

\section{References}

[1] JIANG Shuang-shuang, LIU Peng-yuan, ZHANG Xi'en. Method of Process-Modeling in Virtual Maintenance Based on Petri Net[J]. Journal of System Simulation, 2007, 19(11): 2488-2491.

[2] Zhou Dong,Huo Lin,Wang Meihui,Mao Deyao.Research and application of virtual maintenance technology[J].Joumal of Beijing University of Aeronautics and Astronautics, 2011, 37(2): 231-236.

[3] LI Tai-fu,HUANG Mao-lin,XIE Zhi-jiang. Knowledge Representation for Rotative Machine Fault Diagnosis Based on Semantic Net[J].Journal of Chongqing University(Natural Science Edition), 2001, 24(6): 18-20.

[4] Liu Jiaxue, Liu Tao, Geng Hong. Process modeling and Application in Virtual Maintenance based on Petri Net 
and Semantic Network[J]. JOURNAL OF GRAPHICS,2013,34(2):113-118.

[5] Hu Pan, Jiang Lai. Construction of virtual interactive assembly system[A]. Applied Mechanics and Materials-ICMEAT 2013[C]. Switzerland: rans Tech Publications Ltd,2014. 254-259. 